\title{
Achieving a trusted, reliable, Al-ready infrastructure for military medicine and civilian care
}

Cindy Crump, Loretta Schlachta-Fairchild

Cindy Crump, Loretta M. Schlachta-Fairchild, "Achieving a trusted, reliable, Al-ready infrastructure for military medicine and civilian care," Proc. SPIE 11413, Artificial Intelligence and Machine Learning for Multi-Domain

Operations Applications II, 114130C (21 April 2020); doi: 10.1117/12.2557514

SPIE. Event: SPIE Defense + Commercial Sensing, 2020, Online Only 


\title{
Achieving a Trusted, Reliable, AI-Ready Infrastructure for Military Medicine and Civilian Care
}

\author{
Cindy A. Crump, PhD, PMP, CISSP; Loretta Schlachta-Fairchild, PhD, RN, FACHE, LTC (ret.) \\ U.S. Army Medical Research \& Development Command 1054 Patchel Street, Ft. Detrick, MD, USA
}

21702-3012

\begin{abstract}
It is envisioned that significant improvements in medical capabilities may be required to meet formidable conditions expected in future military conflicts and global events such as the COVID-19 pandemic. Similar challenges may exist for large-scale humanitarian assistance missions and civilian mass casualty events that do not conform to prior assumptions for care delivery including evacuation within the golden hour and availability of large medical footprints in non-traditional and field settings. The importance of standardization and foundational infrastructure for medical devices, sensors, and data management is presented in order to achieve safe, and effective medical systems that deliver dramatic advances in functionality made possible by Artificial Intelligence and Machine Learning (AI/ML). The concept of autonomous, artificial intelligence based learning systems for medical support in military Multi-Domain Operations (MDO) to meet evolving demands is presented. Drivers towards greater use of Artificial Intelligence (AI) and Medical Autonomy to solve anticipated gaps in forward resuscitative and stabilization care, as well as associated relevance and implications for the management of civilian disasters are introduced. Finally, the central role of application architecture and robust technology frameworks necessary to advance the state of the science are discussed.
\end{abstract}

The views, opinions, and/or findings contained in this abstract are those of the authors and do not necessarily reflect the views of the Department of Defense and should not be construed as an official DOD/Army position, policy, or decision unless so designated in other documentation. No official endorsement of any product or vendor is made. Reference here-in to any specific commercial products, process, or service by trade name, trademark, manufacturer, or otherwise, does not necessarily constitute or imply its endorsement, recommendation, or favoring by the U.S. Government.

Keywords: Application architecture, AI Stack, Medical Artificial Intelligence (Medical AI), Medical Robotics and Autonomous Systems (MedRAS), Multi-Domain Operations (MDO), Artificial Intelligence (AI) in Medical Support, Medical Device Interoperability (MDI)

\section{INTRODUCTION}

Recent military conflicts have relied on air superiority and relatively few casualties to support treatment and evacuation within 60 minutes, the so-called "Golden Hour.",

Today, military and civilian medical planners cannot rely on these assumptions and need to prepare for prolonged field care over many days in austere, pre-hospital conditions lacking facilities or infrastructure required for the current state in medical planning, practices and evacuation support. In future conflicts, and prolonged civilian disaster events such as pandemics or hurricanes, limited air access, extended geographic supply lines, and high numbers of casualties may quickly overwhelm available medical and evacuation resources. This gap suggests new solutions and greater efficiencies that can scale quickly while building, and delivering local and regional medical expertise in real-time using ethical, evidence-based AI, medical robotics, and autonomous systems (MedRAS). Artificial intelligence and machine learning (AI/ML) may enable medical systems to automatically learn and improve from experience without being explicitly programmed to achieve a target outcome. AI/ML may be applied to medical devices and patient monitoring data to identify hidden patterns and features in the data supporting automated probabilistic inferences such as predicting the likelihood 
an event will happen, or autonomously providing medical care in the same way that AI/ML can assist in navigating challenging terrain for the first time. "Machine learning focuses on the development of computer

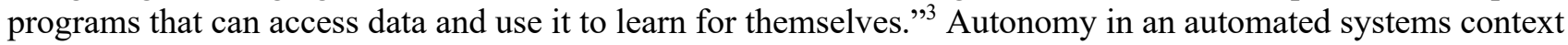
has been described as:

"...the level of independence that humans grant a system to execute a given task. It is the condition or quality of being self-governing to achieve an assigned task based on the system's capabilities such as sensing, perceiving, analyzing, planning, and decision-making required for taking action to achieve an objective." ${ }^{4}$

Robotic and Autonomous Systems (RAS) is considered:

"... an accepted term in academia and the science and technology community and highlights the physical (robotic) and cognitive (autonomous) aspects of these systems. For the purposes of this concept, RAS is a framework to describe systems with a robotic elements, an autonomous element, or more commonly, both."

\section{FUTURE MILITARY MEDICAL CONDITIONS IN MDO}

When support, medical infrastructure (medical devices, patient monitoring data and software systems), and specialty care is either limited, or non-existent, military and civilian first responders in the future may need to rely on semi-autonomous and fully autonomous medical capabilities to provide prolonged or remotely delivered hospital-based or non-traditional/field care to patients. Preventable errors may result from the severe cognitive and physical burdens placed on local healthcare providers when delivering care over extended periods of time. Sustained stressors increase potential risks to patient and healthcare providers' safety.

Just-in-time predictive placement and positioning of medical assets may be needed to assist caregivers in highdemand situations with scarce, and/or rapidly diminishing supplies. In situations like the COVID-19 pandemic, these capabilities could include human-machine collaboration for medical decision-making and advanced lifesupport to sustain patients for several days until evacuation, and/or re-supply becomes possible. Based on local conditions, smaller, more mobile medical units may require rapid expansion, greater self-sufficiency, and/or semi- or fully autonomous medical diagnosis and treatment in advanced systems of care that serve to preserve life and limb under dangerous, dirty, or denied conditions, and in dense, urban environments lacking the infrastructure taken for granted today (including network communications, power and water). More sophisticated cybersecurity measures may be needed to counter ongoing threats to military and civilian medical missions.

\section{ENVISIONING (AND RESEARCHING) THE FUTURE STATE OF MEDICAL CARE FOR MULTI-DOMAIN OPERATIONS}

The use of artificial intelligence (AI) to approximate human judgment at machine speed for a range of clinical applications - from accurate diagnostic image analysis, to patient risk classification, triage, delivery of care and beyond -- is a rapidly advancing field. ${ }^{6,7,8,9}$ Multiple strategic drivers point to the need for autonomous systems that rely on machine learning for delivery of medical capabilities in contaminated or dispersed, farflung settings around the globe. ${ }^{11,12,13.14}$ Early applications to consider, as illustrative examples, include autonomous closed loop control systems that regulate medical devices, patient monitoring data, medications (pain meds/IV fluids, etc.) to automate trauma care for a Combat Medic managing multiple patients, and blood resupply via unmanned aerial and ground vehicles. ${ }^{15}$

The Artificial Intelligence in Medical Sciences (AIMS) Task Force at the US Army Medical Research and Development Command is charged with the development of cross-cutting research that will extend, 
synthesize, and integrate existing Science and Technology (S\&T) lines of effort to close validated military medicine capability gaps by:

1) Identifying and focusing medical research to take advantage of both current and emerging advances in enabling AI technologies that directly address warfighter needs.

2) Establishing strategic, collaborative knowledge-generating research to solve challenging problems in medical operations in a dispersed environment to support the future fight and Warfighter Readiness.

3) Forging Industry, and Academic partnerships as well as new cross-laboratory synergy by sharing software, libraries, tools, methods, and data commons within common frameworks that build and scale expertise and deliver useful, new medical capabilities.

4) Ensuring patient safety by providing ethical, evidence-based medical care that capitalizes on emerging methods of "Assured" Autonomy and AI essential for delivering valid, and trusted clinical inferences across a range of automated medical applications.

An application reference architecture can provide the organizing frame of reference laying the groundwork for reliable, secure, ethical, and evidence-based semi- and fully autonomous medical systems. Sensors and devices that a Warfighter, Combat Medic, or Corpsman carries into battle provide the basic building blocks that enable Warfighter readiness, optimal performance and even delivery of needed care for casualties in austere and dispersed operations. However, today's versions of sensors, medical devices, and systems are often non-standard and vendor proprietary. Such devices are not always interoperable to the level necessary, as demonstrated and available in automated systems within other Industries. Nor are the devices necessarily sufficiently robust to deliver an integrated, yet flexible, and modular set of services required for evolving missions foreseen in MDO.

Medical device interoperability (MDI) is needed but far from reality. Most medical devices have not been designed to be standardized or secure, and some devices on the market today contain significant and potentially dangerous cybersecurity vulnerabilities. ${ }^{16,17}$ The sensors and medical devices required for future military operations, and in global or mass casualty incidents lack an overarching, interoperable, and secure application and data framework. In addition, a cohesive set of standards is required to ensure the level of seamless operation necessary to achieve semi- and fully autonomous care in environments lacking skilled/specialty providers. Open source or standards-based software, and tools for collaboration could provide greater accessibility, tailored to the evolving priorities of battlefield medicine and global medical needs. These elements may support enhanced warfighter and medical readiness, and mission command situational awareness, while minimizing technical support requirements and empowering medical personnel operating under stressful conditions.

\section{AN “AI-READY STACK” FOR ETHICAL, EVIDENCE-BASED HUMAN-MACHINE TEAMING AND MEDICAL AUTONOMY}

Systems engineering approaches specify an ordered method for integrating modular, and flexible subcomponents into highly complex, reliable, and scalable "system of systems" structures that achieve desired automation goals. Industry and academic institutions are advancing the state of the art in AI and autonomous systems through useful systems engineering models such as the concept of an "AI Stack." 18 The stack provides a technical architecture framework composed of interoperable, layered components that abstract and encapsulate complexity. This enables higher order functionality made possible the further one moves up the stack. Interfaces between layers ensure consistency and interoperability - from the lowest elements in the physical layer (computing, sensors) to fully autonomous applications in software that mimic elements of human learning, and decision-making. 
To provide an analogy, the person shown in Figure 1 possesses an array of capacities inherent in human cognition. Judgment is required for successful navigation of the environment. Experience supports and improves decision-making through continuous learning from signals provided by the environment. Humans use sensory perception to collect information, learn from experience, and store prior actions with responses held in memory. This facilitates new decision-making and actions. These separable functions collectively contribute to informed action in novel and evolving situations within a virtuous decision loop.

The AI Stack depicted in Figure 1 provides a conceptual model detailing an orderly and flexible set of components that serve a higher automated system goal or mission. The combined elements support an approximation of the functions of human cognition, i.e. perceive, learn, decide, and act.

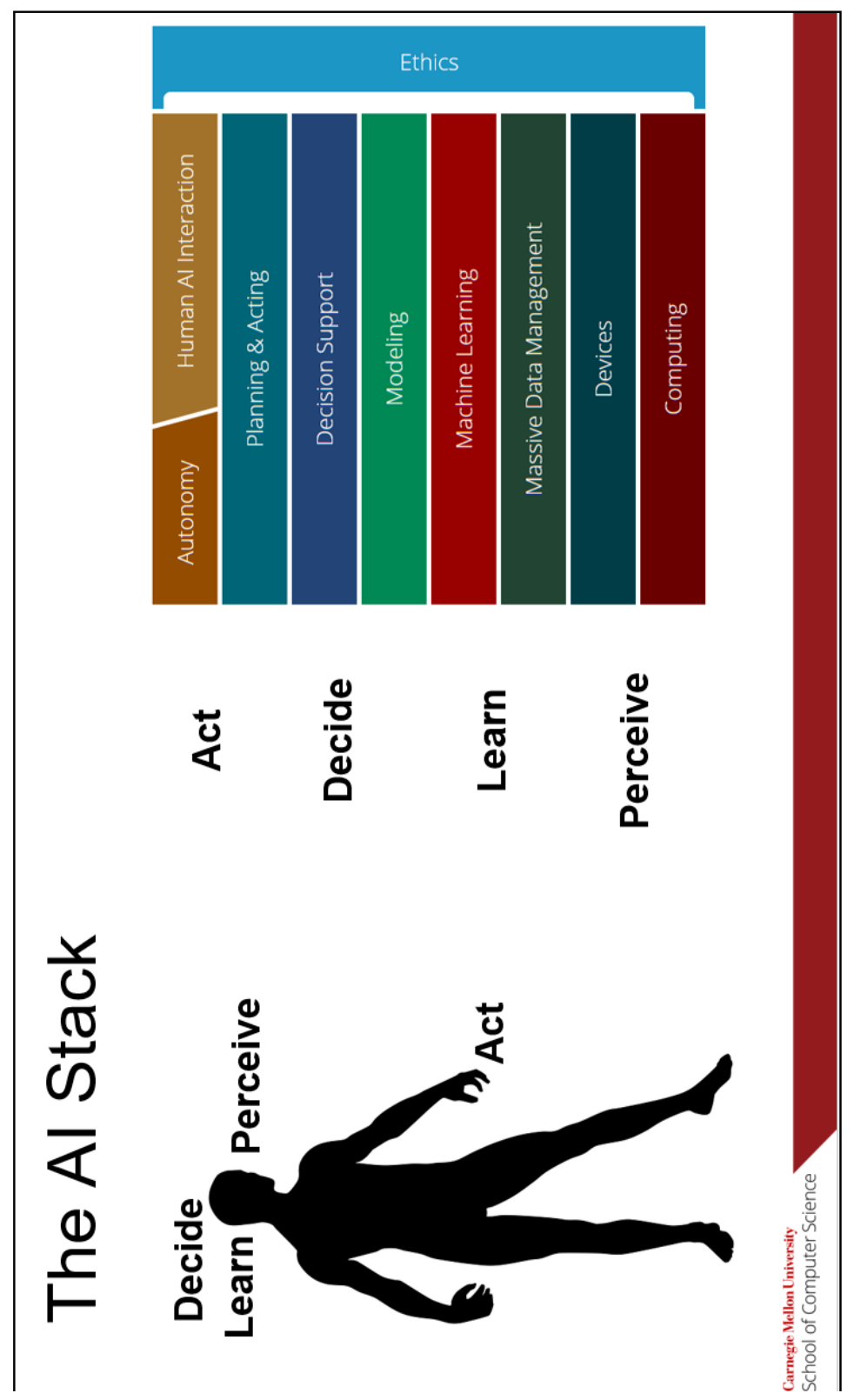

Figure 1: The AI Stack (used with permission by Carnegie Mellon University) 
The AI stack is comprised of layered elements, each contributing to the automated system through the specified function in each layer, enabled by services below. AI and autonomy is achieved through perception, using basic computing and sensor devices to collect and store data from the environment (i.e. operating system, sensors, actuators, and data management). Machine learning tools, modeling/simulation functions, and decision support capabilities support the application which learns from the data available, and plans by modeling probable outcomes for a constrained set of plausible actions. This layered approach culminates in an autonomous system or human / AI interaction that achieves the highest order of functionality. The goal of providing reliable, secure, and robust autonomous systems and human /AI teaming across diverse operational, design and development contexts may be more likely to be achieved through use of a reference architecture. The architecture and ethics, in combination, ensure the system adheres to specified policies that guide safetycritical system design, and operation within appropriate bounds.

The AI Stack model presented in Figure 1 is similar in many respects to the internationally recognized Open Systems Interconnection (OSI) "7-Layer Network Model" from the International Standards Organization (ISO). ${ }^{19}$ The network reference model has advanced the state of the science in network technologies including the Internet. Significant innovation with economic benefits have been realized over the last forty years because the model achieved its original objective of interoperability, and reliability using open standards that have been proven across diverse systems and operating contexts.

Figure 2 depicts the concept of the Medical AI Stack adapted to medical missions supporting automated clinical decision-making and autonomous systems envisioned in MDO. The architectural elements specify a trusted, layered, and open framework for successful medical care using human-machine collaboration. The medical providers 'decision-loop' in this context is approximated through technology components at the lowest layers of computing and devices (sensors, robotic actuators, controls, network, etc.), combined with shareable, high-quality data repositories, libraries, and toolsets that facilitate collaboration and learning. Higher levels of functionality including machine learning, and modeling support the ability of the system to mimic some of the cognitive processes of a healthcare provider. These functions include ability to assess, decide, and ultimately intervene in and conduct autonomous patient care. The architecture bounds the range of possible actions to ensure policy-based, ethical, evidence-based systems that safely deliver valid clinical inferences and outcomes. 


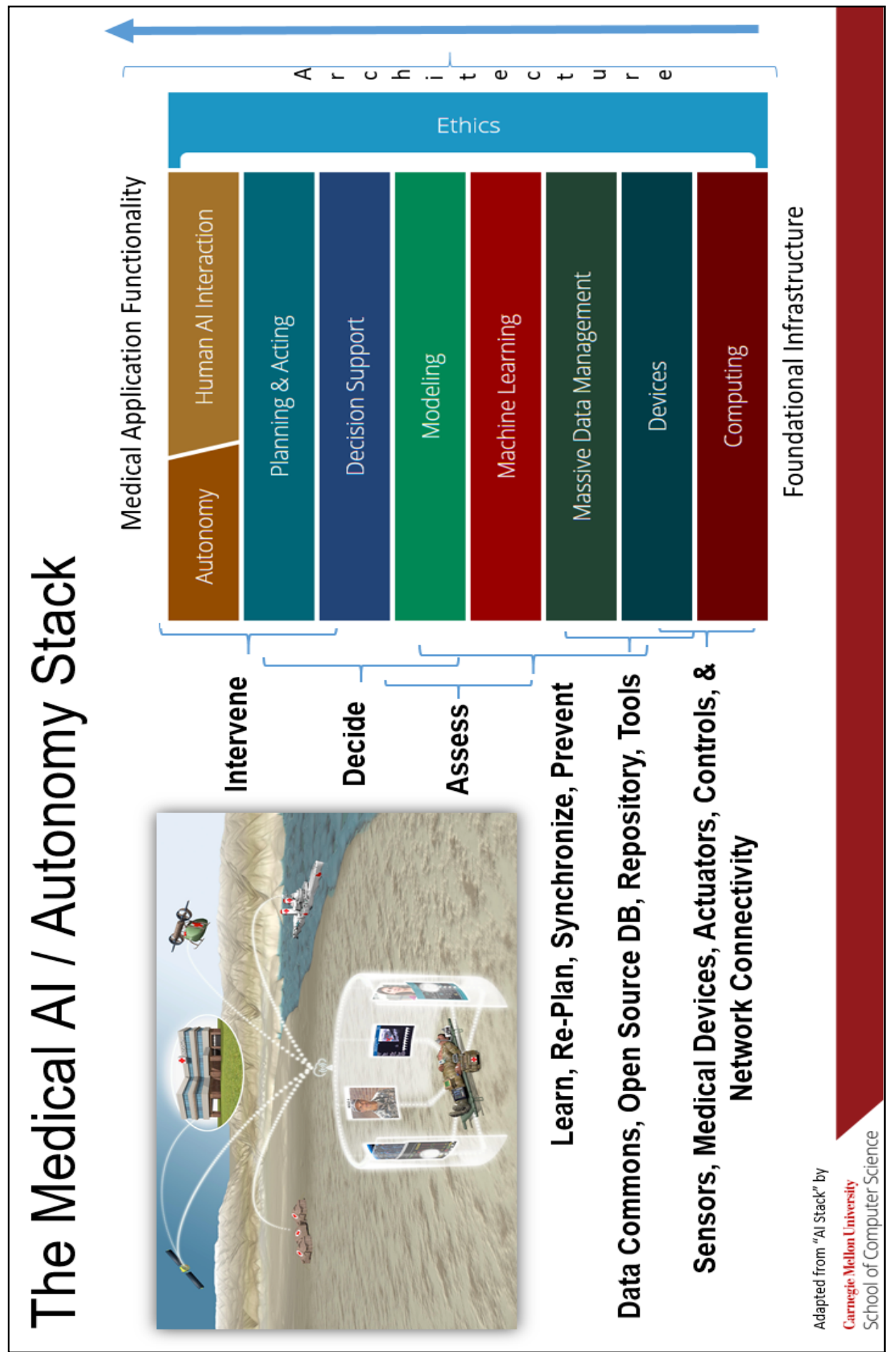

Figure 2: The Medical AI / Autonomy Stack (Adapted with permission of Carnegie Mellon University) 


\section{TACKLING THE STACK ONE LAYER AT A TIME: A MEDICAL DEVICE INTEROPERABILITY REFERENCE ARCHITECTURE (MDIRA) RESEARCH COLLABORATION}

The Defense Health Agency in collaboration with the US Army Medical Research and Development Command is sponsoring research to design and develop a secure, interoperable medical device interoperability reference architecture. ${ }^{1}$ The architecture research collaboration includes an array of Industry and Academic partners as well as Federal Agency participation from the Food and Drug Administration (FDA), and the National Institutes of Standards and Technology (NIST). The research is expected to include design guidance and specifications for secure, and interoperable medical AI/autonomy applications. The applications could include: 1) closed loop control systems of medical care that securely and safely support patient assessment and direct clinical interventions in semi or fully autonomous modes, and 2) AI-assisted clinical decision support.

The central aim of the MDIRA research is to engage the medical device community in broad-based research that fosters not only a standards-based reference architecture, but also reference implementations. The reference implementations are expected to demonstrate future state possibilities and prototypes for Medical $\mathrm{AI}$ and autonomous medical capabilities. Open-source reference models, demonstrations, and running code in collaboration with the community is an expected outcome to encourage adoption of standards-based solutions necessary to advance the state of the science. ${ }^{20}$ For those interested in participating in this open research collaboration, more information, as well as the draft architecture specifications are available for review on the website. $^{21}$

\section{REFERENCES}

[1] Johns Hopkins Applied Physics Laboratory "Medical Device Interoperability Reference Architecture Research Collaboration Video," Youtube, October 23, 2019." https://www.youtube.com/watch?v=aMMbCpNqIiI

[2] Rasmussen, T. E., \& Kellermann, A. L.,"Wartime Lessons — Shaping a National Trauma Action Plan,” The New England Journal of Medicine. 375(17), 1612-1615 (2016).

[3] Varone M, Mayer D., \& Melegari, A. "What is Machine Learning? A Definition," Expert System https://www.expertsystem.com/machine-learningdefinition/ (2019).

[4] Congressional Research Service, "U.S. Ground Forces Robotics and Autonomous Systems (RAS) and Artificial Intelligence (AI): Considerations for Congress." https://crsreports.congress.gov/product/pdf/R/R45392 p. 2 (1 November 2018).

[5] Ibid.

[6] Hinton, G. " Deep Learning - A Technology With the Potential to Transform Health Care." JAMA, 320(11) (2018).

[7] Maddox, T. M., Rumsfeld, J. S., \& Payne, P. R. O, "Questions for Artificial Intelligence in Health Care," Journal of the American Medical Association, 321(1), 31-32, https://doi.org/10.1001/jama.2018.18932 (2019).

[8] Shah, N. H., Milstein, A., \& Bagley PhD, S. C., "Making Machine Learning Models Clinically Useful," JAMA Français. Chicago, IL : https://doi.org/10.1001/jama.2019.10306 (2019).

[9] Sim, I., "Mobile Devices and Health," New England Journal of Medicine, 381(10), 956-968. https://doi.org/10.1056/nejmra1806949 (2019).

[10] Stead, W., "Clinical Implications and Challenges of Artificial Intelligence and Deep Learning," JAMA, 320(11). Retrieved from http://search.proquest.com/docview/2116408195/ (2018).

[11] Fisher, N., \& Gilbert, G., "Medical Robotic and Autonomous System Technology Enablers for the Multi-Domain Battle 2030-2050," Small War Journal. (2017).

[12] Joint Chiefs of Staff. (2015). "Joint Concept for Health Services,"

https://www.jcs.mil/Portals/36/Documents/Doctrine/concepts/joint_concept health services.pdf?ver=2017-12-28$\underline{162027-633}(2015)$. 
[13] Congressional Research Service, "U.S. Ground Forces Robotics and Autonomous Systems (RAS) and Artificial Intelligence (AI): Considerations for Congress." https://crsreports.congress.gov/product/pdf/R/R45392 (1 November 2018).

[14] U.S. Training and Doctrine Command. "Multi-Domain Battle: Evolution of Combined Arms for the 21st Century 2025-2040," https://www.tradoc.army.mil/Portals/14/Documents/MDB_Evolutionfor21st (1).pdf (2017).

[15] Schlachta-Fairchild, L., Crump, C., Gilbert, G., Little, J., Salinas, J., Benante, J. P., .. Bills, M. (2019). Medical Autonomous Systems for Improving Healthcare Delivery. Proc. AUVSI XPONENTIAL 2019,

https://www.xponential.org/xponential2019/public/SessionDetails.aspx?FromPage=Speakers.aspx\&SessionID $=3138 \& n a$ $\mathrm{v}=$ true\&Role $=\mathrm{U} \% 27$ (2019).

[16] Health Care Industry Cybersecurity Task Force. "Report on Improving Cybersecurity in the Health Care Industry," https://www.phe.gov/Preparedness/planning/CyberTF/Documents/report2017.pdf (2017).

[17] Klonoff, D., \& Han, J. (2019). The First Recall of a Diabetes Device Because of Cybersecurity Risks. Journal of Diabetes Science and Technology, 13(5), 817-820. https://doi.org/10.1177/1932296819865655

[18] Carnegie Mellon University School of Computer Science, “AI Stack," (2019).

[19] Weik, M. H., "OSI Reference Model-The ISO Model of Architecture for Open Systems Interconnection," Computer Science and Communications Dictionary, C(4), 1146-1146. https://doi.org/10.1007/1-4020-0613-6 12825 (2006).

[20] Goldman, J. “Medical Device "Plug-and-Play" Interoperability Program,” https://www.mdpnp.org (2020).

[21] Johns Hopkins University Applied Physics Laboratory "Medical Device Interoperability Reference Architecture Specifications," https://secwww.jhuapl.edu/mdira (2020). 